\title{
Serotype-specific detection of African horsesickness virus by real-time PCR and the influence of genetic variations
}

\author{
J.J.O. Koekemoer* \\ Onderstepoort Veterinary Institute, Private Bag X05, and Department of Veterinary Tropical Diseases, University of Pretoria, Private Bag X04, Onderstepoort 0110, South Africa
}

\section{Article history:}

Received 9 June 2008

Received in revised form 11 August 2008

Accepted 15 August 2008

Available online $\mathrm{xxx}$

\section{Keywords:}

African horsesickness

Serotype

Real-time RT-PCR

Hybridization probes

\begin{abstract}
A B S T R A C T
Real-time PCR hybridization probe sets were tested for the specific detection of amplified genome segment 2 cDNA from all nine serotypes of African horsesickness virus (AHSV). The hybridization probes were derived from the sequences of genome segments 2 of the nine reference strains of the virus and were designed to have clearly distinguishable peak melting temperatures. Viral dsRNA from each of the serotypes was specifically detected after reverse transcription, real-time PCR and melting curve analysis. The method was used to successfully serotype a range of field isolates, although most of the these showed peak melting temperature shifts. These shifts could be related to nucleotide substitutions in the regions that are targeted by the probes. Sensitivity was demonstrated to be sufficient for use with dsRNA isolated directly from infected organ samples, making it potentially useful as a rapid diagnostic tool.
\end{abstract}

(c) 2008 Elsevier B.V. All rights reserved.

\section{Introduction}

African horsesickness (AHS) is a non-contagious, arthropod borne infection of Equidae and possibly other animals (Coetzer and Erasmus, 1994). Clinical signs vary but in naive horses it causes a rapidly progressing disease that is fatal in most cases. AHS is caused by infection with the African horsesickness virus that is a member of the genus Orbivirus in the family Reoviridae. The virus species consists of nine antigenic types that have been identified using panels of neutralizing antisera (McIntosh, 1958; Howell, 1962) and have been designated AHSV serotypes 1-9.

The disease is endemic in most of tropical and sub-tropical Africa and is of great economic importance because of its typical high mortality and rapid spread during so-called "horsesickness seasons". In South Africa, a number of recent outbreaks have severely constrained local and international movement of horses for trade and other purposes. Outside Africa, epizootics have been experienced in countries around the middle East (Rafyi, 1961) and in Europe (Lubroth, 1988; Rodriguez et al., 1992). Fears that AHS might reemerge outside sub-Saharan Africa have been heightened (Mellor and Hamblin, 2004) following the incursion and unprecedented persistence of bluetongue, a related vector borne viral disease of ruminants, into Western Europe in 1998 (Baylis, 2002). This is especially feasible considering that the horse populations are not protected by vaccination. It is generally accepted that the move-

\footnotetext{
* Tel.: +27 12 5299229; fax: +27 125299304

E-mail address: KoekemoerO@arc.agric.za.
}

ment of infected host animals plays the major role in the spread of AHS but investigators have been speculating that the Culicoides sp. vector, infected with either of the related orbiviruses, bluetongue virus (BTV) or epizootic hemorrhagic disease virus, could be spread over considerable distances by prevailing winds (Sellers and Maarouf, 1991; Alba et al., 2004; Gloster et al., 2007).When immunization is used to control specific outbreaks, especially in non-endemic areas where monovalent vaccines are used, it is essential to know the serotype against which to immunise. Serotyping of AHSV is classically done using virus neutralization tests but this is a time consuming process that requires virus isolation and cell culture techniques. Genome segment 2 codes for VP2 and even before large scale nucleotide sequencing had been carried out, it was shown that this part of the genome is highly divergent among the nine serotypes of AHSV (Bremer et al., 1990). This has since been confirmed after sequencing of genome segment 2 of all the serotypes (Potgieter et al., 2003). A logical conclusion was to develop serotype-specific molecular tests that identify nucleotide sequences on genome segment 2 . These have been reported in the form of serotype-specific RT-PCR primers (Sailleau et al., 2000) and genome segment 2 serotype-specific probe hybridization (Koekemoer et al., 2000; Koekemoer and Van Dijk, 2004). With the latter method it was shown that there is enough variance between the nine serotypes for probes, consisting of partial genome segment 2 amplification products, to hybridize in a serotype-specific manner.

This work describes the development of this concept into a real-time PCR method by designing a panel of nine serotypespecific hybridization probe sets and demonstrating their ability to

0166-0934/\$ - see front matter @ 2008 Elsevier B.V. All rights reserved. doi:10.1016/j.jviromet.2008.08.010 
Table 1

Sequence and predicted peak $T_{\mathrm{m}}$ of hybridization probe sets

\begin{tabular}{|c|c|c|c|}
\hline Serotype & $T_{\mathrm{m}}\left({ }^{\circ} \mathrm{C}\right)$ & Anchor probe & Sensor probe \\
\hline 1 & 51.2 & CCGTATTTGGGTGAAA-FAM & LC640-TATTTTTCGCCGGAGAACTAT-PO4 \\
\hline 2 & 55.9 & ATGACAAGCGATTGATGAAA-FAM & LC640-TAAAATTCAGCCGTATATGGGTGAAA-P04 \\
\hline 3 & 52.1 & TATGAGAAAGAGATGTGTGA-FAM & LC705-TAATTATTACAGCGGAGAATGCA-PO4 \\
\hline 4 & 57.5 & AGATGTGAGGGGGCAT-FAM & LC640-CCAATTTTTCCGCGTTATATAATTGATACG-PO4 \\
\hline 5 & 56.9 & CAGGCGATTCAAATGAATGT-FAM & LC705-AGATGCTGAGAGCGTTTGTACC-PO4 \\
\hline 6 & 58.0 & ATTTTACCGCACTATGTCACAGAC-FAM & LC705-CATTAAATATGGGATGGTAATCGATAG-PO4 \\
\hline 7 & 59.4 & TATGAGTGGGGCGCAAC-FAM & LC640-TCACCGATTAGGACTATGCGAAATAGA-PO4 \\
\hline 8 & 59.7 & TATGAGTGGGAGTTTACAGACCATAG-FAM & LC705-TGGGGCTCTGCGAAGAGAG-PO4 \\
\hline 9 & 63.2 & GCAGCCGTACTTAGGTGATGC-FAM & LC640-ATTTTTCACCAGAGCATTACACCGCGA-PO4 \\
\hline
\end{tabular}

specifically detect cDNA from the nine serotypes of AHSV. The probes were also tested using field isolated viruses of all nine serotypes and with dsRNA isolated from clinical samples in an effort to evaluate their usefulness in diagnostic applications.

\section{Materials and methods}

\subsection{Viruses and cells}

All the field viruses were isolated and serotyped at the Onderstepoort Veterinary Institute (OVI) from samples submitted for AHSV testing from cases in Southern Africa. The reference strain viruses that were used have been described by Potgieter et al. (2003). The field viruses are listed in Table 3 and were all isolated by inoculation of equine blood or homogenized organ samples into suckling mouse brains followed by three passages on BHK cell cultures. After isolation the viruses were kept as freeze-dried stocks. Serotyping was done through standard virus neutralization assays. With both the reference and field strain viruses, Vero cell cultures propagated in $75 \mathrm{~cm}^{2}$ flasks were infected with freeze-dried virus stocks reconstituted in $1 \mathrm{ml}$ of medium and harvested when clear CPE was visible through $80-100 \%$ of the culture. This seed material was used to infect subsequent Vero cell cultures for dsRNA preparation.

\section{2. dsRNA preparation}

Viral RNA was extracted from infected cells with a commercial acid-phenol/guanidinium thiocyanate based reagent (TRI-reagent, Molecular Research Centre, Inc.) as described before (Koekemoer and Van Dijk, 2004). dsRNA was isolated by means of differential LiCl-precipitation. Between 100 and $200 \mu$ l of homogenized spleen tissue was used for the preparation of dsRNA from infected organ samples. The RNA was extracted with $1000 \mu \mathrm{l}$ of TRI-reagent and the rest of the process was the same as that used for infected cell culture material.

\subsection{Probe design}

A universal set of RT-PCR primers (Koekemoer and Van Dijk, 2004) was used to amplify the first 521-553 base pairs of genome segment 2 of all the reference and field isolate viruses. Nine sets of hybridization probes were designed to each hybridize only with target cDNA from the complementary AHSV serotype and to have a unique peak $T_{\mathrm{m}}$ that could be identified by a melting curve analysis. dsRNA from the nine reference strain viruses were used to determine serotype-specificity and the peak $T_{\mathrm{m}}$ of the nine probe sets. The nine hybridization probe sets each consisted of one anchor and one sensor probe and were designed from the sequences of the nine reference strain viruses using the LightCycler ${ }^{\circledR}$ Probe design software ver. 2.0 (Roche Diagnostics). The probes were designed to bind to genome segment 2 in such a way that the $3^{\prime}$-end of the anchor probe will be in close proximity to the $5^{\prime}$-end of the sensor probe. The $3^{\prime}$-ends of the anchor probes were labeled with 6 -Carboxyfluorescein (FAM) and the $5^{\prime}$-ends of the reporter probes with either LC640 or LC705. Binding of the two probes next to each other on the target cDNA will result in fluorescence resonance energy transfer (FRET) between the FAM and LC640 or LC705 dyes upon excitation of the FAM dye. FRET will then result in the emission of a light signal at 640 or $705 \mathrm{~nm}$, depending on the LC dye on the sensor probe. GenBank accession numbers for the reference strain sequences are: AHSV1: AY163329; AHSV2: AY163332; AHSV3: U01832; AHSV4: AHVCP2A; AHSV5: AY163331; AHSV6: NC_005996; AHSV7: AY163330; AHSV8:AY163333 and AHSV9: AF043926. The nine sets of probes were designed to make differentiation possible on two levels: alternative reporter dyes, LC640 and LC705, were used to label the sensor probes and probe sets with the same reporter dye were designed to have clearly distinguishable peak melting temperatures $\left(T_{\mathrm{m}}\right)$. Table 1 gives details of the hybridization probes that were used.

\subsection{Reverse transcription and real-time $P C R$}

Reverse transcription was carried out on dsRNA isolated from infected cell cultures or organ samples. The dsRNA was denatured with $\mathrm{MMOH}$ and RT was initiated with genome segment 2 universal primers as described before (Koekemoer and Van Dijk, 2004). One microliter of the cDNA mixture was used to perform the realtime PCR in a final volume of $20 \mu l$. The real-time PCR mixture consisted of the LightCycler®FastStart DNA Master ${ }^{\text {PLUS }}$ HybProbe master mix (Roche Diagnostics), $0.1 \mu \mathrm{M}$ of each of the universal segment 2 primers (Koekemoer and Van Dijk, 2004) and $0.2 \mu \mathrm{M}$ each of the anchor and sensor probes (Table 1). The hybridization probes were manufactured by TIB MOLBIOL GmbH. A pre-PCR incubation at $95^{\circ} \mathrm{C}$ was carried out to activate the hot-start polymerase followed by 35-cycles of PCR in a LightCycler 1.5 real-time PCR instrument using the following cycling parameters: $95^{\circ} \mathrm{C}$ denaturing for $5 \mathrm{~s}, 45^{\circ} \mathrm{C}$ annealing for $10 \mathrm{~s}$ and extension at $72^{\circ} \mathrm{C}$ for $22 \mathrm{~s}$. As the universal RT-PCR is not the serotype-specific step in the method, a low primer annealing temperature could be used to provide optimal sensitivity without the concern that non-specific amplification would influence the specificity of the result. Fluorescence was measured in the 640 and $705 \mathrm{~nm}$ channels after each annealing step and software based colour compensation was applied to prevent bleed over of fluorescence between the channels.

\subsection{Melting curve and crossing point analysis}

After the PCR was completed, a melting curve analysis was carried out in three steps: all the probes and template cDNA were melted at $95^{\circ} \mathrm{C}$ after which probe hybridization was allowed for $30 \mathrm{~s}$ at $45^{\circ} \mathrm{C}$. The temperature was increased to $80^{\circ} \mathrm{C}$ at a rate of $0.2^{\circ} \mathrm{C}$ per second with continuous measurement of fluorescence. The values for crossing points $(\mathrm{CP})$ and peak $T_{\mathrm{m}}$ are given as reported 
by the LightCycler Software 4.0 (Roche Diagnostics). The CP value is the fraction of the PCR cycle number at which the logarithmic phase of the reaction begins as determined by a maximum in the increase of fluorescence.

\section{Results}

\subsection{Serotype-specificity}

All the probe sets hybridized only to cDNA amplified from virus dsRNA of the corresponding serotype using the parameters described above for the real-time PCR. Table 2 gives the CP and peak $T_{\mathrm{m}}$ values as reported by the software for each of the serotypes. No amplification signals ( $\mathrm{CP}$ values) were reported where probes were tested with non-homologous serotypes, indicating serotypespecificity in all cases.

\subsection{Melting curve analysis}

The nine sets of hybridization probes were designed to have clearly distinguishable peak $T_{\mathrm{m}} \mathrm{s}$ in two different detection channels
Table 2

$\mathrm{CP}$ and peak $T_{\mathrm{m}}$ values derived from hybridization probe detection of reference strain AHSV cDNA

\begin{tabular}{lll}
\hline Serotype & CP value & Peak $T_{\mathrm{m}}\left({ }^{\circ} \mathrm{C}\right)$ \\
\hline 1 & 15.43 & 53.26 \\
2 & 17.87 & 62.87 \\
3 & 17.39 & 58.01 \\
4 & 17.59 & 60.68 \\
5 & 16.37 & 60.34 \\
6 & 18.64 & 64.01 \\
7 & 13.47 & 57.87 \\
8 & 16.87 & 67.93 \\
9 & 12.45 & 69.32 \\
\hline
\end{tabular}

(Table 1) with the possibility of multiplexing the probes in mind. Each of the two probes that make up a hybridization probe pair has a specific $T_{\mathrm{m}}$. The lower of the two temperatures was used to predict the peak $T_{\mathrm{m}}$ of the probe set as release of either the anchor or sensor probes would cause a decrease in fluorescence. On the basis of different peak melting temperatures and the wavelength of the emitted light signal it would therefore be possible to identify and distinguish one or more specific probe sets that hybridized to a
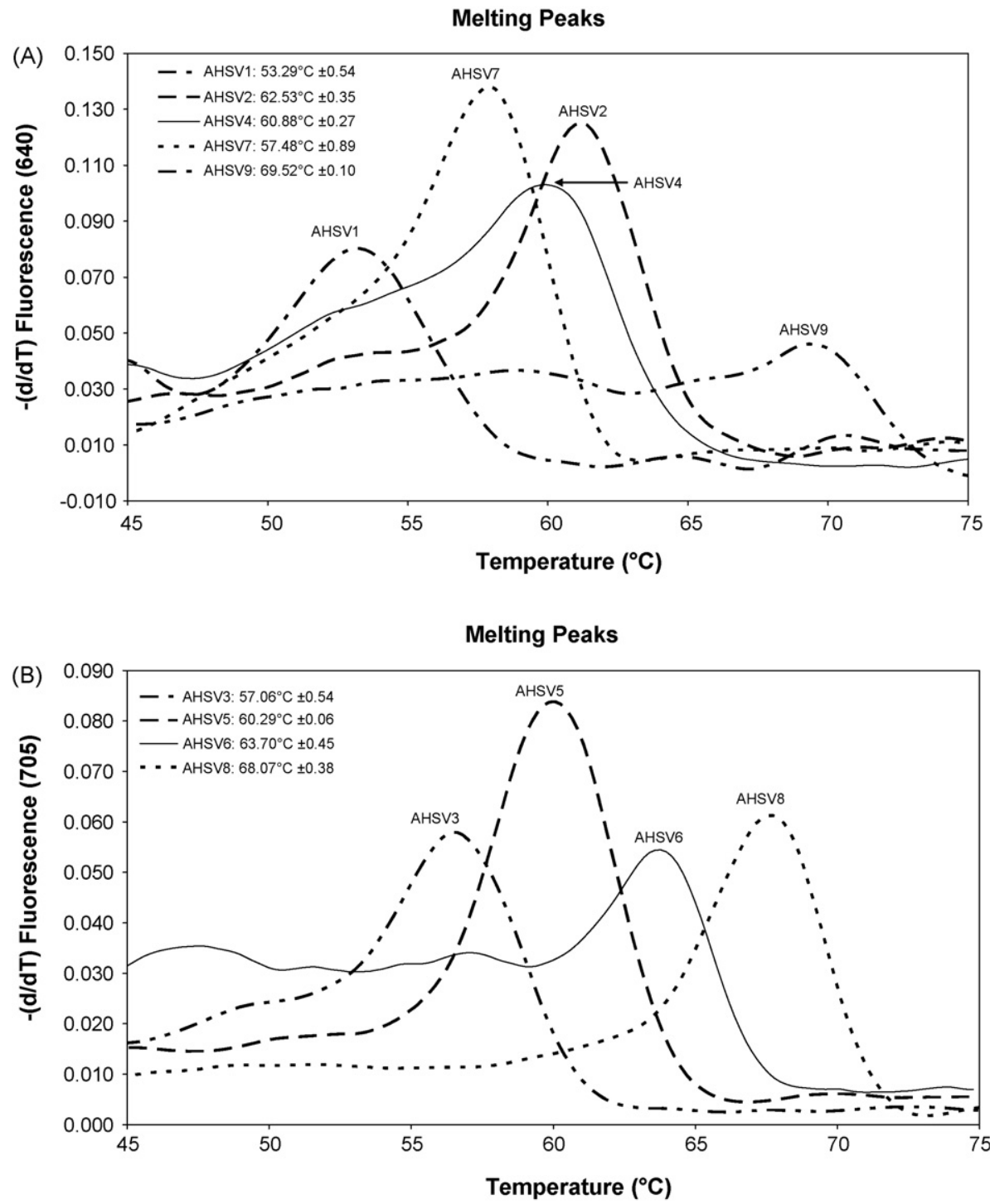

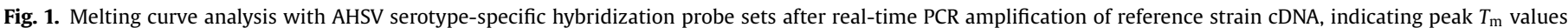
measured (A) in the $640 \mathrm{~nm}$ and (B) in the $705 \mathrm{~nm}$ channels. 
homologous cDNA target(s) when the probes are multiplexed. Fig. 1 shows the results from melting curve analyses that were carried out after the real-time PCR was completed. The five melting peaks in channel 640 (Fig. 1A) and the four in channel 705 (Fig. 1B) were all clearly distinguishable. Between run variations were low after replicate testing with different dsRNA preparations of the reference strain viruses and it did not interfere with probe identification by this means. Mean $T_{\mathrm{m}}$ values with the standard deviation for five replicates are indicated on Fig. 1.

\subsection{Intra-serotype variation of peak $T_{m}$}

The hybridization probe sets were tested using a range of field viruses of all nine serotypes (Table 3 ) to investigate the effect of possible intra-serotype nucleotide sequence variations in the probe binding areas on melting curve analyses. Identification of amplified cDNA by measurement of real-time fluorescence was serotypespecific in all cases. Marked peak $T_{\mathrm{m}}$ variations were, however, observed with all but the serotype 1 field isolates (Table 3). This was an indication of sequence variations in the areas targeted by one or both of the probes and it was decided to confirm this by sequencing. Isolates showing the highest deviation from the reference strain peak $T_{\mathrm{m}}$ were sequenced and Fig. 2 shows the alignments with indications of nucleotide substitutions that were found in field isolates.

\subsection{Duplexing hybridization probes}

The variation in genome segment 2 limits multiplexing to combinations of probes that do not fluoresce at the same wavelength. Duplex probe sets reporting in alternate channels were therefore considered for multiplexing. Selections were based on $\Delta G$ (free energy) calculations of possible cross hybridizations between pairs of probe sets (data not shown). On this principle the following probe set pairings were selected: AHSV 1 with AHSV 3, AHSV 2 with AHSV 8, AHSV 4 with AHSV 5 and AHSV 6 with AHSV 9. The AHSV 7 probe set was not tested in combination with other
Table 3

AHSV field viruses used for peak $T_{\mathrm{m}}$ testing with serotype-specific hybridization probes

\begin{tabular}{llllll}
\hline Isolate & Serotype & Peak $T_{\mathrm{m}}\left({ }^{\circ} \mathrm{C}\right)$ & Isolate & Serotype & Peak $T_{\mathrm{m}}\left({ }^{\circ} \mathrm{C}\right)$ \\
\hline HS13/99 & 1 & 53.06 & HS33/05 & 5 & 54.41 \\
HS197/06 & 1 & 53.22 & HS185/06 & 5 & 55.52 \\
HS170/06 & 1 & 53.29 & HS4/98 & 6 & 54.83 \\
HS125/08 & $\frac{1}{2}$ & $\underline{53.17}$ & HS26/03 & 6 & 54.76 \\
HS11/97 & 2 & 61.11 & HS194/06 & 6 & 53.91 \\
HS9/03 & 2 & 59.53 & HS45/03 & 7 & 56.81 \\
HS20/03 & 2 & 59.47 & HS34/03 & 7 & 56.56 \\
HS217/06 & 2 & 59.84 & HS56/04 & 7 & 56.81 \\
HS157/06 & 2 & 59.77 & HS89/06 & 7 & 56.96 \\
HS50/08 & 2 & 58.13 & HS87/08 & $\underline{7}$ & $\underline{7}$ \\
HS14/98 & 3 & 47.55 & HS119/08 & $\underline{7}$ & $\underline{57.25}$ \\
HS44/02 & 3 & 56.87 & HS120/08 & $\underline{7}$ & $\underline{57.28}$ \\
HS09/02 & 3 & 47.66 & HS129/08 & $\underline{7}$ & $\underline{57.46}$ \\
HS10/03 & 3 & 47.04 & HS17/98 & 8 & 62.25 \\
HS55/08 & $\frac{3}{3}$ & $\underline{47.45}$ & HS29/00 & 8 & 61.31 \\
HS74/08 & $\underline{4}$ & $\underline{47.12}$ & HS83/04a & 8 & 47.60 \\
HS25/98 & 4 & 56.39 & HS6/01 & 9 & 51.20 \\
HS28/03 & 4 & 56.59 & HS168/06 & 9 & 50.25 \\
HS128/06 & 4 & 48.57 & HS203/06 & 9 & 50.00 \\
HS188/06 & 4 & 48.39 & HS211/06 & 9 & 50.19 \\
HS3/06 & 5 & 55.83 & & & \\
\hline
\end{tabular}

The year of isolation is given by the last two digits in the isolate name.

a All viruses were isolated from South-African samples except for HS83/04 from Namibia and HS203/06 from Botswana. Underlined enteries indicate viruses that were typed directly from dsRNA from spleen samples.

probes. Hybridization probes were used at a final concentration of $2 \mu \mathrm{M}$ each and tested with three cDNA template combinations: one with cDNA from the first serotype, one with cDNA from the second serotype and one containing both. In all cases cDNA from the reference strain viruses was used. All serotypes could be detected by real-time fluorescence signals when using the mixed probe sets. Where cDNA from both serotypes were included, both could be detected as amplification signals of the two hybridization probe sets in alternate fluorescence measuring channels. Fig. 3 gives the results of AHSV 1 and 3 probes used in duplex with

\begin{tabular}{|c|c|}
\hline AHSV2 & ATCAGGATGACAAGCGATTGATGAAAATTAAAATTCAGCCGTATATGGGTGAAATGTATTT \\
\hline $\mathrm{HS} 20 / 03$ & ATCAGGACGACAAGCGATTGATGAAAATTAAAATTCAGCCGTATATGGGCGAAATGTATTT \\
\hline AHSV3 & АTCAAACATATGAGAAAGAGATGTGTGATGTAATTATTACAGCGGAGAATGCAGTTAGAA \\
\hline $\mathrm{HS} 10 / 03$ & АTCAAACATATGAGAAAGAGAAGTGCGATGTAATTATTACAGCGGAAAATGCAGTTAGAA \\
\hline AHSV4 & TGAGATCAGATGTGAGGGGGCATATCCAATTTTTCCGCGTTATATAATTGATACGTTAAAA \\
\hline HS188/06 & TGAGATCAGGTGTGAGGGAGCATATCCAATTTTTCCGCGTTATATAATCGATACGTTAAAA \\
\hline AHSV5 & GAAGAGACAGGCGATTCAAATGAATGTTGAGATGCTGAGAGCGTTTGTACCAAAAAGG \\
\hline HS33/05 & GAAGAGACAAGCGATTCAAATGAATGTTGAAATGCTGAGAGCATTTGTGCCAAAAAGG \\
\hline HS185/06 & GAAGAGACAAGCGATTCAAATGAATGTTGAAATGCTGAGAGCATTTGTACCAAAAAGG \\
\hline AHSV6 & AGGAGACTAACTATAGGTTAGGATTGTGCGAAATAGAGAATACGATGTCAATCAGTGATTT \\
\hline HS194/06 & AAGAGACTAATCATAGATTGGGATTGTGCGAAATAGAAAATACGGTATCAATCAGTGĀTT \\
\hline AHSV7 & GGGTATGAGTGGGGCGCAACAAATCACCGATTAGGACTATGCGAAATAGAACACGTAAAGA \\
\hline HS56/04 & GGGTATGAGTGGGGCGCAACAAATCATCGATTAGGGCTATGCGAAATAGAACACGTGAAGA \\
\hline AHSV8 & AAAGGCTATGAGTGGGAGTTTACAGACCATAGGCTGGGGCTCTGCGAAGAGAGTTATCTG \\
\hline HS29/00 & AAAGGCTATGAGTGGGAATTTACAGACCATAGGCTGGGGCTCTGCGAAGAGAGTTATCTG \\
\hline HS83/04 & AAAGGCTATGAGTGGGAATTTACAGATCATAGGCTWGGGCTCTGCGAAGAGAGTTATCTG \\
\hline AHSV9 & ACAAGTGCAGCCGTACTTAGGTGATGCCTATTTTTCACCAGAGCATTACACCGCGACCTTCTT \\
\hline HS203/06 & ACAAGTACAGCCGTATTTAGGCGACGCTTATTTTTCGCCAGAGTATTACACTGCTACCTTCTT \\
\hline HS211/06 & ACAAGTACAGCCGTATCTAGGCGACGCTTATTTTTCGCCAGAGTATTACCCCGCTACCTTCTT \\
\hline
\end{tabular}

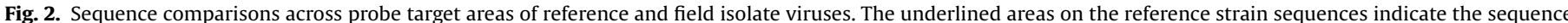
of the hybridization probe pairs and shaded nucleotides show the extent of substitutions that occur in field isolates. 

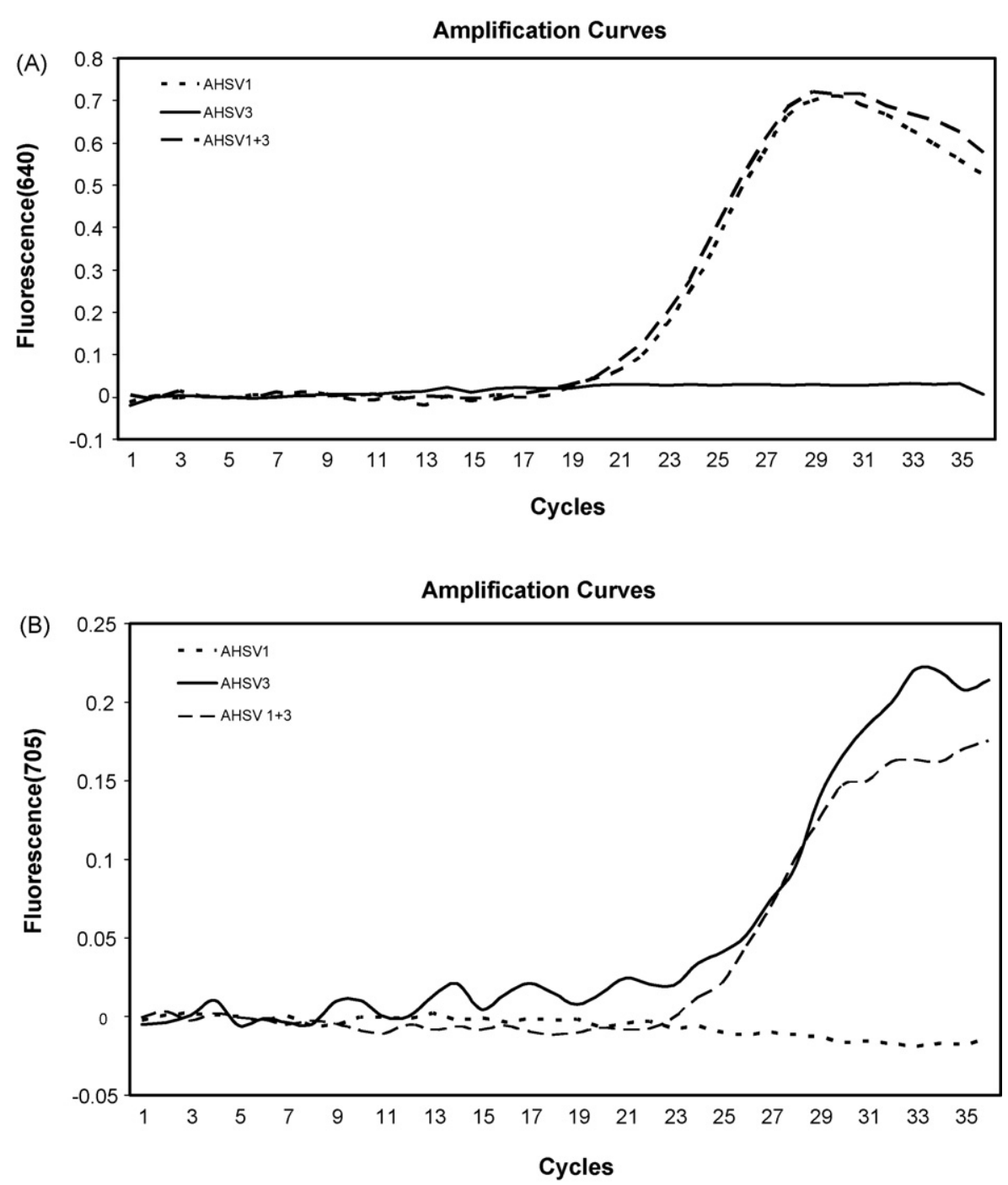

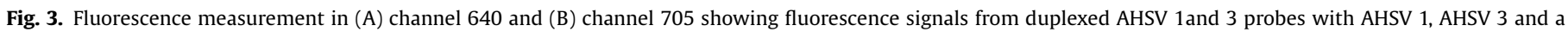
mixture of AHSV $1+3$ represented by the three lines respectively.

combinations of AHSV serotypes 1 and 3. The identity of the specific probe set that hybridized was determined from the channel (640 or 705) in which fluorescence was detected above the threshold by a CP value. Similar results were obtained for the other probe combinations. No fluorescence was observed when nonhomologous serotypes were tested with any of the duplex probe sets.

\subsection{Sensitivity}

To determine the limit of sensitivity, different amounts of AHSV 2 and AHSV 5 dsRNA were prepared as dilution series and used for RT and real-time PCR with the serotype 2- and 5-specific hybridization probes included at $0.4 \mu \mathrm{M}$. The dilution series started with a total of $1.0 \mathrm{ng} / \mu \mathrm{l}$ of AHSV dsRNA and ended with a $10^{-6}$ dilution in water $(1.0 \mathrm{fg} / \mu \mathrm{l})$. In each case $5 \mu \mathrm{l}$ of the particular dilution was used. Amplification and peak melting temperatures were measured in channel 640 for AHSV 2 and channel 705 for AHSV 5 (Table 4). In both cases the $10^{-4}$ dilution, containing 5 pg of dsRNA, was the limit of detection as determined by qualitative detection of amplification after 40 cycles.

\subsection{Serotype determination from organ samples}

AHSV dsRNA from infected organ samples were also used to determine the suitability of the method to be used in a rapid diagnostic application. RNA was isolated from post mortem spleen samples that were collected from suspect cases and submitted for laboratory confirmation of AHS. Eight samples of four serotypes were tested and the results from the real-time PCR method was in accordance with serological typing that was carried out on the

Table 4

CP and peak $T_{\mathrm{m}}$ values obtained after RT and real-time PCR detection of two dilution series of AHSV dsRNA

\begin{tabular}{ccccccc}
\hline Total amount of dsRNA/pg & \multicolumn{2}{l}{ AHSV2 } & & \multicolumn{2}{l}{ AHSV5 } \\
\cline { 2 - 3 } \cline { 6 - 7 } & CP & Peak $T_{\mathrm{m}}\left({ }^{\circ} \mathrm{C}\right)$ & & $\mathrm{CP}$ & Peak $T_{\mathrm{m}}\left({ }^{\circ} \mathrm{C}\right)$ \\
\hline 5000 & 19.77 & 62.76 & & 17.70 & 60.59 \\
500 & 23.19 & 62.97 & & 23.75 & 60.76 \\
50 & 27.61 & 63.01 & & 31.37 & 60.90 \\
5.0 & 29.12 & 62.84 & & $>35.00$ & 59.63 \\
0.5 & - & - & & - & - \\
0.05 & - & - & & - & - \\
\hline
\end{tabular}


viruses that had previously been isolated from the same organs. The typing results when using dsRNA isolated from infected organ samples are underlined in Table 3.

\section{Discussion}

Rapid and sensitive diagnostic tests remain the most important tool for confirming and monitoring AHS outbreaks. The traditional way of confirming AHS is by virus isolation from clinical specimens that are submitted for testing. After the virus is isolated in cell culture, it is serotyped using immunological procedures. The biggest obstacle in this process is virus isolation that can in many cases not be achieved and takes up to 2 weeks to complete. The serotype of an AHSV can be determined indirectly using techniques that characterize segment 2 of the virus genome. One such a method, which is sensitive enough to be used directly on the dsRNA isolated from clinical specimens, has been described (Koekemoer and Van Dijk, 2004). It entails RT-PCR amplification of cDNA of the $5^{\prime}$-end of genome segment 2 which is then characterized by hybridization with probes on a solid support membrane. This paper describes an advancement of the method and was developed to eliminate labour intensive membrane hybridization procedures and replaces it with a real-time PCR that uses hybridization probes. These probe pairs make use of the FRET-principal to provide a fluorescence signal upon duplex forming of the two adjacent probes with a stretch of target DNA (Heller and Morrison, 1985; Cardullo et al., 1988). It is one of the most useful real-time PCR techniques and has the advantage that a post PCR melting curve analysis can be carried out. This makes probe discrimination possible without the need for specific labels (Wittwer et al., 2001). Furthermore, hybridization probes will reveal nucleic acid substitutions by changes in the peak melting temperature of the probe sets and it is often used as a sensitive genotyping tool (Bernard and Wittwer, 2000). In the field of orbivirus diagnostics this sensitivity has been applied in probe based real-time PCR tests that can go as far as distinguishing between different strains of single serotypes of BTV (Orrù et al., 2004; Elia et al., 2008).

The method of amplifying cDNA from of the 5 '-end of genome segment 2 with a universal primer set was used unchanged as it proved to be a reliable and very sensitive way of providing cDNA for serotype-specific hybridization (Koekemoer and Van Dijk, 2004). The genome segments 2 sequences of the prototype viruses (Potgieter et al., 2003) were used for the design of the hybridization probe sets. When dsRNA from these reference viruses were tested, increased fluorescence signals, that indicate cDNA amplification and probe hybridization, were serotype-specific in all cases. Although the peak $T_{\mathrm{m}}$ varied with up to $8.23^{\circ} \mathrm{C}$ from what was predicted by the design software (Tables 1 and 2) the values were clearly distinguishable and could be used to identify each of the serotypes (Fig. 1). The serotype of reference strain viruses could repeatedly be determined from melting curve analysis alone, supporting the idea of probe multiplexing. This changed when dsRNA preparations from field viruses were used (Table 3 ). The results showed varying probe peak $T_{\mathrm{m}}$ values as a result of nucleotide substitutions in the area where the probes hybridized. From previous sequence analyses of genome segment 2 of AHSV 7 (Koekemoer et al., 2003) and AHSV 2 (unpublished data) field isolates, these variations were known to be a possibility but their numerous appearance in the short (42-51 bp) hybridization probe targets was not predicted. Other workers have reported similar problems with BTV identification using real-time PCR (Jiménez-Clavero et al., 2006) and this phenomenon is something that is warned against when using melting curve analysis as a diagnostic tool (Whiley and Sloots, 2005). Sequence variation in the probe target site occurred in 10 out of 48 bases when comparing the refer- ence and one of the field isolates of AHSV 9 (Fig. 2). This was the highest variance that was observed and probably stems from the fact that the AHSV 9 reference strain (HS 90/61) was isolated in 1961 from the middle East (information from AHSV reference centre, OVI) while the field viruses are recent isolates from Southern Africa. Notwithstanding the base pair mismatches, (Fig. 2) the probes hybridized specifically to genome segment 2 cDNA from matching serotypes, making serotype determination possible by qualitative detection of fluorescence increase. During the annealing step of the PCR the temperature was decreased to $45^{\circ} \mathrm{C}$ (the optimal temperature for the universal primers) allowing probe hybridization to the template cDNA. The serotypes of all the field isolates determined in this fashion corresponded with serotyping results from virus neutralization or partial genome segment 2 sequencing.

As expected, the intra-serotype sequence variation that was observed in the VP2-gene was highest between the sequence of prototype viruses and that of the most recent field isolates. Sequence variation among isolates of one serotype has been found to be lower when there is less time separation ( 1 or 2 years) between isolation and then it seems to be more related to geographic separation (Koekemoer et al., 2003). The prototype strains were all isolated during 1961-1963 and low passage stocks were used to obtain the genome segment 2 nucleotide sequences (Potgieter et al., 2003) that were used to design the probes. Except for the serotype 1 isolates, all the field viruses that were used showed decreased peak $T_{\mathrm{m}}$ values when tested. These viruses are all recent isolates (1997-2008) and could all be expected to have accumulated nucleotide substitutions in the probe target areas, causing the peak $T_{\mathrm{m}}$ shifts. Some of these sequence variations were confirmed by sequencing and it led to the conclusion that serotype identification by probe peak $T_{\mathrm{m}}$ would not be possible. This limited the multiplexing of probes to two pairs that fluoresce at different wavelengths. During duplexing, probe identification was therefore based on the emitted wavelength of the fluorescence signal and not on its peak $T_{\mathrm{m}}$. The combination of two hybridization probe sets had no effect on the specificity of hybridization as determined by fluorescence increase measurements. It is foreseen that the number of probe sets that can be multiplexed could be increased by using real-time PCR instruments that can measure fluorescence in more than two channels. This would be necessary considering the possibility of designing hybridization probes that could discriminate between closely related genotypes of the same serotype, increasing the number of probes to be multiplexed. Identification of vaccine strain viruses (as has been reported for BTV by Orrù et al., 2004; Elia et al., 2008) is one obvious application. In its present form the serotype-specific real-time PCR would not be able to distinguish between different strains, including the vaccine viruses, of any particular serotype of AHSV. This could be seen as a drawback as the vaccine viruses could be present in diagnostic samples, especially from endemic areas.

The analytical sensitivity of this real-time PCR test was found to be limited to $5 \mathrm{pg}$ of total dsRNA. This is in the same order of magnitude as that of the reverse blot serotype-specific hybridization (Koekemoer and Van Dijk, 2004) and the RT-PCR test that uses serotype-specific primers (Sailleau et al., 2000). Sensitivity is less than what is reported for the group-specific real-time tests for AHSV (Agüero et al., 2008) and BTV (Jiménez-Clavero et al., 2006). These methods, however, make use of more sensitive $5^{\prime}$ Taq nuclease-3'-minor groove binder-DNA probes that hybridize to smaller amplification products. Sensitivity could undoubtedly be increased by re-designing the reverse primers to amplify a shorter part of genome segment 2 . Based on available data the sensitivity appears to be adequate to identify the serotype of AHSV from clinical samples without the need for virus isolation. This is important 
as virus isolation is usually the limiting step in turn-around time of diagnostic results.

In conclusion, these data demonstrate the feasibility of using hybridization probes in a real-time PCR format to determine the serotype of AHSV. As with other orbiviruses, the genetic variation that appears over time makes the design and use of a single set of diagnostic real-time PCR probes to identify all possible genetic variants of the nine AHSV serotypes impossible. Although it was found that hybridization probes will tolerate several nucleotide substitutions, it is suggested that probes are designed based on regularly updated sequence data from current field isolate viruses. Given its specificity the probe repertoire could be expanded to identify not only serotypes but also specific genotypes of interest for diagnosis and epidemiological studies.

\section{Acknowledgements}

The author wishes to thank the Directorate of Veterinary Services of the Gauteng Provincial Government for funding, Dr. Christiaan Potgieter for the 2006/7 AHSV field virus dsRNA and the AHS reference centre at OVI for field isolates and organ samples.

\section{References}

Agüero, M., Gómez-Tejedor, C., Angeles Cubillo, M., Rubio, C., Romero, E., JiménezClavero, A., 2008. Real-time fluorogenic reverse transcription polymerase chain reaction assay for detection of African horsesickness virus. J. Vet. Diagn. Invest. $20,325-328$.

Alba, A., Casal, J., Domingo, M., 2004. Possible introduction of bluetongue into the Balearic Islands, Spain, in 2000, via air streams. Vet. Rec. 155, 460-461.

Baylis, M., 2002. The re-emergence of bluetongue. Vet. J. 164, 5-6.

Bernard, P.S., Wittwer, C.T., 2000. Homogeneous amplification and variant detection by fluorescent hybridization probes. Clin. Chem. 46, 147-148.

Bremer, C.W., Huismans, H., van Dijk, A.A., 1990. Characterization and cloning of the African horsesickness virus genome. J. Gen. Virol 71, 793-799.

Cardullo, R.A., Agrawal, S., Flores, C., Zamecnik, P.C., Wolf, D.E., 1988. Detection of nucleic acid hybridization by non-radiative fluorescence resonance energy transfer. Proc. Natl. Acad. Sci. U.S.A. 85, 8790-8794.

Coetzer, J.A.W., Erasmus, B.J., 1994. African horsesickness. In: Coetzer, J.A.W., Thomson, G.R., Tustin, R.C. (Eds.), Infectious Diseases of Livestock with Special Reference to Southern Africa, vol. 1. Oxford University Press, Cape Town, pp. 460-475.

Elia, G., Savini, G., Decaro, N., Martella, V., Teodori, L., Casaccia, C., Di Gialleonardo, L., Lorusso, E., Caporale, V., Buonavoglia, C., 2008. Use of real-time RT-PCR as a rapid molecular approach for differentiation of field and vaccine strains of bluetongue virus serotypes 2 and 9. Mol. Cell. Probes 22, 38-46.
Gloster, J., Mellor, P.S., Manning A.J., Webster, H.N., Hort, M.C., 2007. Assessing the risk of windborne spread of bluetongue in the 2006 outbreak of disease in northern Europe. Vet. Rec. 160, 54-56.

Heller, M.J., Morrison, L.E., 1985. Chemiluminescent and fluorescent probes for DNA hybridization systems. In: Kingsbury, D.T., Falkow, S. (Eds.), Rapid Detection and Identification of Infectious Agents. Academic Press, New York, pp. 345-356.

Howell, P.G., 1962. The isolation and identification of further antigenic types of African horsesickness. Onderstepoort J. Vet. Res. 29, 139-149.

Jiménez-Clavero, M.A., Agüero, M., San Miguel, E., Mayoral, T., López, M.C., Ruano, M.J., Romero, E., Monaco, F., Polci, A., Savini, G., Gómez-Tejedor, C., 2006. High throughput detection of bluetongue virus by a new real-time fluorogenic reverse transcription-polymerase chain reaction: application on clinical samples from current Mediterranean outbreaks. J. Vet. Diagn. Invest. 18, 7-17.

Koekemoer JJ.O. Paweska, JT, Pretorius, PJ van Dijk, A.A., 2003. VP2 gene phylogenetic characterization of field isolates of African horsesickness virus serotype 7 circulating in South Africa during the time of the 1999 African horsesickness outbreak in the Western Cape. Virus Res. 93, 159-167.

Koekemoer, J.J.O., Potgieter, A.C., Paweska, J.T., Van Dijk, A.A., 2000. Development of probes for typing African horsesickness virus isolates using a complete set of cloned VP2-genes. J. Virol. Methods 88, 135-144.

Koekemoer, J.J.O., Van Dijk, A.A., 2004. African horsesickness virus serotyping and identification of multiple co-infecting serotypes with a single genome segment 2 RT-PCR amplification and reverse line blot hybridization. J. Virol. Methods 122, 49-56.

Lubroth, J., 1988. African horsesickness and the epizootic in Spain 1987. Equine Pract. $10,26-33$.

McIntosh, B.M., 1958. Immunological types of horsesickness virus and their significance in immunization. Onderstepoort J. Vet. Res. 27, 465-538.

Mellor, P.S., Hamblin, C., 2004. African horsesickness. Vet. Res. 35, 445-466.

Orrù, G., Santis, P.D., Solinas, F., Savini, G., Piras, V., Caporale, V., 2004. Differentiation of Italian field and South African vaccine strains of bluetongue virus serotype 2 using real-time PCR. J. Virol. Methods 122, 37-43.

Potgieter, A.C., Cloete, M., Pretorius, P.J., van Dijk, A.A., 2003. A first full outer capsid protein sequence data-set in the Orbivirus genus (family Reoviridae): cloning, sequencing, expression and analysis of a complete set of full-length outer capsid VP2 genes of the nine African horsesickness virus serotypes. J. Gen. Virol 84, 1317-1326.

Rafyi, A., 1961. Horsesickness. Bull. Off. Int. Epizoot. 56, 216-250.

Rodriguez, M., Hooghuis, H., Castano, M., 1992. African horsesickness in Spain. Vet. Microbiol. 33, 129-142.

Sailleau, C., Hamblin, C., Paweska, J.T., Zientara, S., 2000. Identification and differentiation of the nine African horsesickness virus serotypes by RT-PCR amplification of the serotype-specific genome segment 2. J. Gen. Virol. 81, 831-837.

Sellers, R.F., Maarouf, A.R., 1991. Possible introduction of epizootic hemorrhagic disease of deer virus (serotype 2) and bluetongue virus (serotype 11) into British Columbia in 1987 and 1988 by infected Culicoides carried on the wind. Can. J. Vet. Res. 55, 367-370.

Whiley, D.M., Sloots, T.P., 2005. Melting curve analysis using hybridisation probes: limitations in microbial molecular diagnostics. Pathology 37, 254-256.

Wittwer, C.T., Herrmann, M.G., Gundry, C.N., Elenitoba-Johnson, K.S., 2001. Real-time multiplex PCR assays. Methods 25, 430-442. 